\title{
Formalization of target and assessment of quality of the cyber- physical control systems for emissions reduction technologies
}

\author{
Remir Solnitsev ${ }^{1, *}$, and Gennady Korshunov ${ }^{2,3}$ \\ ${ }^{1}$ CAD dept. Saint Petersburg Electrotechnical University "LETI" ul. Professora Popova 5, 197376 St. Petersburg, Russian Federation \\ ${ }^{2}$ IIQS dept. Saint-Petersburg State University of Airspace Instrumentation, 67, Bolshaya Morskaya St., Saint-Petersburg, 190000, Russian \\ Federation \\ ${ }^{3}$ CPSC high school, Peter the Great St. Petersburg Polytechnic University, 29, Polytechnicheskaya St.,Saint-Peterburg, 195251, Russian \\ Federation
}

\begin{abstract}
Monitoring and inventory is a necessary but not sufficient condition to reduce emissions in the energy and industry. The ultimate task of controlling air purification technologies in combination with monitoring is effectively achieved while minimizing the human factor. The creation and application of cyber-physical technology management systems provide a solution to a set of related environmental problems. In addition, the development of models based on cyber-physical systems forms new requirements for the development of technologies for their creation. A special feature of such technologies is close interaction between computing methods with design and production facilities. These issues have not been sufficiently reflected in publications. The article presents the target setting for the creation of cyber-physical technology control systems based on local laws of preservation and the decomposition of the target into macroscopic quality assessments. A mathematical model and methods of quality assessment at the life cycle stages of such systems based on recalculation of probability densities of a priori data are presented. Examples of cyber-physical emission reduction systems in the energy and industry developed under the nature-technology concept are given.
\end{abstract}

\section{Introduction}

Problems and tasks in the air quality problem area include monitoring and management of harmful emissions. Relevant emission monitoring and inventory programs and projects have been established both at the global and regional level [1], and for specific energy and industrial facilities. Well-known publications are usually limited by references to the application of cyber-physical monitoring results for following technologies $[2,3]$. Monitoring is necessary, but the use of cyber-physical systems (CPS) also involves feedback control tasks. Correct and rational penetration into the physical environment using methods and tools of cybernetics must be accompanied by an assessment of the reactions of the physical environment during its control. In the environment, such ultimate objectives are minimization, neutralization, recycling and other problem and objectoriented air quality assurance technologies. In this article, it is proposed that the target of air quality assurance should be presented in local forms on the basis of conservation laws. Air quality assurance technologies are proposed to be implemented at the life cycle stages of the CPS for control of the achievement of the required values of quality indicators based on the two-level model of the specification characteristics and ensuring their parameters. The article proposes to evaluate the quality of systems based on the main characteristics of the requirements specification at the stages of the life cycle. During the design, construction and technological preparation stages of production, there are all kinds of deviations from the limits of established norms and tolerances, having a random character. The article suggests an approach to formalization and decomposition of the CPS objective, a mathematical model and a method for determination (recalculation) of the density distribution of probability characteristics and parameters at any point in time on given initial a priori data. When recalculating parameters at this stage, not only time can be taken into account, but also the functions of dynamic errors, inconsistencies in changes in parameters and characteristics of technological processes.

\section{Materials and methods}

The developing class of systems called CPS is characterized by deep penetration and close, including feedback, connection of methods and means of cybernetics and physical environment [6, 9]. The physical environment is subject to research and monitoring for the following control of its facilities. Emissions from energy and industrial units can be fully represented in the physical environment of the CPS.

* Corresponding author: remira70@mail.ru 
Moreover, the purpose of the CPS as a necessary attribute of any control system can be formulated with sufficient certainty, including on the basis of objective conservation laws [10]. At the same time, the implementation of the life cycle of the CPS in relation to the above objects is related to the presence of uncertainties and random events. The implementation of the development, design and technological preparation stages of production should take into account random factors to ensure the quality of the CPS. The article consistently considers the approach to formalization of the target based on the models of physical processes and their interpretation in terms of control and formal representation of the target in the form of probability functionality of the requirements of the specification (quality indicators). Further decomposition of the target to the level of parameters that form these quality indicators allows to move to probabilistic quality estimates based on given initial a priori data - probability densities.

\subsection{Formalization of the CPS target}

The target of the CPS in the form of reducing emissions in the energy and industry through technology management can be formulated on the basis of physical laws that summarize many experiments and describe the evolution of the sought quantities, both in space and time. Most problems in physics lead to the need to solve differential equations[11]. It was shown in [4] that the equation of turbulent diffusion and convection corresponds to the character of the emission processes. This equation represents the local form of the law of conservation of mass and, after transformations, is reduced to the "input-output" form necessary for the implementation of the control functions for emission reduction technologies. "Output" is the emissions that are mitigated by the technology control system. The system, in turn, is subject to requirements, the fulfillment of which is represented by the generalized functional of the "maximum probability of quality assurance" [12] including the probability $P$ of meeting the requirements of the technical task (TT) $\Phi_{0}=\max P\left(V_{0}, W_{0}\right)$, where $V_{o}$ and $W_{0}$ are "initial" characteristics and criteria. At subsequent stages, they are coordinated up to the "n-th" stage of production preparation.

$$
\begin{aligned}
& \Phi=\max P\left[K_{1} \geq K_{1_{0}}, K_{2} \geq K_{2_{0}}, \ldots, K_{j} \leq K_{j_{0}},\right. \\
& \left.K_{j+1} \leq K_{(j+1)_{0}}, \ldots, K_{n} \leq K_{n_{0}}\right]
\end{aligned}
$$

where $\{K j\}, 1 \leq j \leq n$, are TT requirements, including basic requirements (quality characteristics).

\subsection{Decomposition of the target into macro and micro levels}

The target in the CPS is decomposed into macroscopic characteristics that serve to assess the quality. Behind these basic characteristics of the CPS there are many different parameters, which are responsible for numerous designers and manufacturers of individual subsystems, units and parts of the CPS.

The quality of the CPS is determined by compliance with the requirements. The main characteristics, called here "macroscopic", should not go beyond the limits established by the TT. At the stages of the life cycle, nonconformities are assessed in the form of deviations and errors. Macroscopic assessments at the stages of the life cycle, including the processes of design and production of CPS, are carried out on the measured coordinates (variables) $Y(t)=\left(y_{i} \ldots y_{n}\right)$. These variables depend both on time and on many other arguments $Y\left(t, \lambda_{1}, \lambda_{2}, \ldots, \lambda_{n}\right) \quad$ where $\lambda_{i}-$ are the parameters of the FSC (mass, moments of inertia, inductance, capacitance, element materials). Parameters, in the general case, have a probabilistic nature of the "movement of parameters" $\lambda_{i}$, similar to the microscopic movement of particles in a macroscopic volume, when macroscopic characteristics are measured - indicators of the quality of the entire filled volume.

In contrast to the above analogy of "physical filling", the measured characteristics of macroscopic variables $Y\left(t, \lambda_{1}, \lambda_{2}, \ldots, \lambda_{n}\right)$ at the stages of the CPS life cycle depend not only on the parameters of the object itself, but also on the parameters of the design and production process. This is especially true for the stages of technological preparation of production and testing and selection of technological and testing equipment. At these stages, the "developer enterprise" receives from the "manufacturer" notifications about changes in design documentation. These notifications are often spontaneous and random.

\subsection{Assessment of characteristics}

Deviations from TT and errors have the character of random functions, the ordinates of which go beyond the established norms and tolerances. In the theory of random processes, this is reduced to the so-called "outlier problem", the solution of which does not lend itself to correlation theory.

The proposed approach is reduced to solving the following problems.

Determination in the design process of the probability of the ordinates of the measured variables going beyond the limit values of the TT and the duration of exceeding these values in these cases, correlation analysis is inapplicable and it is necessary to obtain the density of probability distributions in time and in time itself. This is due to the fact that these probabilistic characteristics at time $t_{k+s}$ are not related to characteristics at time $t_{k}$. Assessments obtained in the early stages of design should provide an assessment of possible product defects in production.

Assessment of the state of technological equipment in preparation for production and in the production process.

Monitoring the state of the "project" during its movement from the technical assignment to the 
installation according to the probabilistic characteristics of the output variables.

Reduction of labor costs for testing by correcting design documentation and improving the technological preparation of production.

When solving these problems, it is proposed to proceed from the representation of the CPS as a dynamic system with the components $Y(t)=\left(y_{i} \ldots y_{n}\right)$ and $\lambda\left(\lambda_{i} \ldots \lambda_{m}\right)$ as a random function (RF) and a random variable (RV). The most complete characteristics of physical (technical, economic, social) dynamics processes with variables $Y(t)$ and parameters $\lambda$ $Y=\left(y_{i} \ldots y_{n}\right), \quad \lambda\left(\lambda_{i} \ldots \lambda_{m}\right)-\mathrm{RF}$ and $\mathrm{SV}$ are their probability distribution densities $-P_{y}\left(y_{i} \ldots y_{n}, t\right)$ and $P_{\lambda}\left(\lambda_{i} \ldots \lambda_{n}\right)$. On the other hand, the state of the life cycle processes of the CPS as a dynamic system is represented in the form of a vector equation:

$$
Y=F(Y, \lambda, \mathrm{t}), Y_{0}
$$

For further transformations, you need to go to the new variables and parameters $-P_{y}$ and $P_{\lambda}$.

When considering at the beginning a particular case, when $\lambda$ are deterministic, and the initial conditions $Y_{0}$ are random values, the initial probability density $P_{y, 0}\left(y_{10}, \ldots, y_{n 0}, t_{0}\right) . P_{y}$ and $P_{y, 0}(y, 0)$ satisfy the valuation conditions $\int_{-\infty}^{\infty} \ldots \int_{-\infty}^{\infty} P\left(y_{i}, \ldots, y_{n}, t\right) d y_{i} \ldots d y_{n}=1$. To solve problems at all stages, it is desirable to find $P_{y}(Y, \lambda, \mathrm{t})$ at an arbitrary moment of time according to a given initial a priori characteristic $P_{y, 0}\left(Y, \lambda, \mathrm{t}_{0}\right)$.

\subsection{Assessment of quality at the life cycle stages}

At the initial stages, it is advisable to develop an enlarged metamodel of the product, according to which a differential equation is constructed for the new variables $P_{\mathrm{y}}$. In the future, this model is transformed into intermediate mathematical models [12].

The initial $P_{0}\left(Y, \lambda, \mathrm{t}_{0}\right)$ is set according to a priori data obtained from previous studies, analogs and results of a preliminary analysis of design problems, accumulated in databases [13...15]. In particular, if it is possible to obtain statistical characteristics obtained at the stages of testing elements or according to the data of the manufacturer of component parts, then the "recalculation" of the probability densities of these data in $P_{\lambda}$ will allow setting $P_{y, 0}\left(Y, \lambda, \mathrm{t}_{0}\right)$, where $\lambda$ is given as a random variable.

The arguments for RF and $Y$ are not necessarily time. They can be represented by ordinates, functions of dynamic errors, inconsistencies in parameter changes, characteristics of technological processes.The transition from the system of equations (2) to new variables leads to a partial differential equation of the form

$$
\frac{d P}{d t}=(Y, \lambda, t)-\frac{d P}{d Y} F(Y, \lambda, t)+P \frac{d P}{d Y}=0, P_{0}(Y, \lambda, t)
$$

Equation (2) in symmetric form takes the form

$$
\frac{d t}{1}=\frac{d Y}{F(Y, \lambda, t)}=\frac{d P(Y, \lambda, t)}{\mathrm{P}(Y, \lambda, t) \frac{d F(Y, \lambda, t)}{d Y}}
$$

Assuming that Eq. (4) determines $P(Y, \lambda, \mathrm{t})$ at any moment of time, which, through the Jacobi matrices $d P / d Y$ and $d F / d Y$, are functions of the parameters $\lambda$, the probability function $\Phi(<Y<,<\lambda<,<t<)$ are introduced. These functions can be interpreted as assessments of the area of operability of the future product at the appropriate stages of the life cycle.

Based on the solution of equations (4), the probability of the variable $Y(t)$ going beyond the given corridor (the problem of emissions) during the interval $\mathrm{T}$ is estimated, $T-\Phi\left[a<y(t)<b\left|t_{0}<t<T\right| \lambda_{\text {min }}<\lambda<\lambda_{\text {max }}\right]$ If this solution is found, then $\Phi=\int_{a}^{b} \int_{t_{0}}^{T} P_{y}(y, \lambda, t) d y d t$. Assuming the application of the normal distribution law of the probability density $P_{y}(Y, \lambda, \mathrm{t})$ at each given moment of time $\mathrm{t}$, according to the known relations, the probabilities of fulfilling the established norms are found for the main measured characteristics

$$
\Phi=\frac{1}{\sqrt{2 \pi \sigma}} \int_{a}^{b} e-\frac{\left(y_{k}-\bar{y}\right)^{2}}{2 \sigma^{2}} d y=\Phi_{1}\left(\frac{b-\bar{y}}{\sigma}\right)-\Phi_{2}\left(\frac{a-\bar{y}}{\sigma}\right)(5)
$$

Where $\Phi_{1}, \Phi_{2} \quad-\quad$ tabular functions $y_{k}=y\left(t_{k}\right), \overline{\mathrm{y}}$ mathematical expectation.

The average duration of the ejection, $\bar{\tau}$, is estimated by the well-known formulas of the theory of random functions. Estimates using formulas similar to formula (4) are carried out throughout the selected life cycle and represent the probability of achieving the goal formulated as functional (1).

The recalculation of the parameters obtained at these stages of the life cycle into the estimated characteristics is carried out on the basis of the known in the theory of the probability of the relationship $P_{z}=P_{x}\left[\varphi^{-1}(x)\right]\left[\varphi^{-1}(x)\right]$ ', where $P_{z^{-}}$is the probability density of the parameter $z, P_{x}$ is the known probability density of the parameter $x, z=\varphi(x)$ is the given design characteristic.

\section{Results}

The results obtained make it possible to obtain probabilistic assessments of the quality of systems according to the main characteristics of the technical specifications at the stages of the life cycle. In this case, various kinds of deviations from the boundaries of the established norms and tolerances, which are random in 
nature, can be taken into account when performing the stages of design, construction and technological preparation of production. The proposed mathematical model makes it possible to determine the probability density distribution of characteristics and parameters at an arbitrary moment in time for a given initial a priori characteristic. When recalculating the parameters at the stage under consideration, not only time can be taken into account, but also the functions of dynamic errors, inconsistencies in parameter changes, and characteristics of technological processes.

\section{Discussion}

The tasks of obtaining a priori information remain relevant. Their solution may be associated with the creation and accumulation of the content of knowledge bases in the relevant problem areas.

\section{Conclusion}

The given approach, mathematical models and methodology make it possible to ensure the quality control of CPS, the characteristic feature of which is the uncertainty of behavior and the random nature of the processes. The materials in this article are not intended to be complete and will be developed for receiving a priori data, a detailed description of the life cycle and in practical applications.

\section{References}

[1] Executive Body for the Convention on Long-range Transboundary Air Pollution [Electronic resource], Thirty-ninth session, Geneva (9-13 December 2019) Available at: https://emep.int

[2] T. Sanislav, G. Mois, S. Folea, L. Miclea, G. Gambardella, P. Prinetto, Proceedings of the 3ed Mediterian Conference on Embedded Computing MECO, Montenegro, Budva, 6-9 (2014)

[3] V.N. Ruchkin, B.V. Kostrov, A.N. Kolesnikova, V.A. Fulin, V.V. Drozdov, Cyber-physical monitoring of natural and man-made environment, Bulletin of the Tula State University, Technical Science 09 (2016)

[4] R.I. Solnitsev, G.I. Korshunov, Control systems «nature-technogenics», Politekhnika, SanktPetersburg (2013) (In Russian)

[5] G.I. Korshunov, R.I. Solnitsev, IOP Conference Series: Earth and Environmental Science 87, 4, 042008 (2017)

[6] E.A. Lee, S.A. Seshia, Introduction to Embedded Systems, A Cyber-Physical Systems Approach, Second Edition, MIT Press (2017)

[7] I. Ruchkin, Integration of Modeling Methods for Cyber-Physical Systems, PhD thesis, Institute for Software Research School of Computer Science, Carnegie Mellon University, Pittsburgh, CMU-ISR18-107 (2019)
[8] E. Lee, Sensors, Basel 15 4837-4869 (2015)

[9] E. Lee, A Fundamental Limits of Cyber-Physical Systems Modeling ACM Tr. on Cyber-Physical Systems (2016)

[10] G.I. Korshunov, S.L. Polyakov, J. Phys.: Conf. Ser. 1515, 2, 022065 (2020)

[11]A.N. Tikhonov, A.A. Samarsky, Equations of mathematical physics (Moscow, Nauka, 1977)

[12] R.I. Solnitsev, G.I. Korshunov, Science and business: ways of development $\mathbf{6}, 108,96-100$ (2020)

[13] Y. Du, Y. Guo, Technology and Health Care 23, s1, S161-S167 (2015)

[14] R.I. Solnitsev, D.X. Cho, Programmnye Producty i Sistemy 101, 125-129 (2013)

[15] R.I. Solnitsev， N.G. Ryzhov, G.I. Korshunov, D.X. Cho, A.V. Paranichev, Proceedings of 2017 20th IEEE International Conference on Soft Computing and Measurements, SCM 2017, 7970681, 662-665 (2017) 